\title{
Intrapelvic Dislocation of a Femoral Trial Head During Primary Total Hip Arthroplasty Requiring Laparotomy for Retrieval
}

\author{
Mustafa Citak ${ }^{*}, 1$, Till Orla Klatte ${ }^{1}$, Akos Zahar ${ }^{1}$, Kimberly Day ${ }^{1}$, Daniel Kendoff ${ }^{1}$, Thorsten Gehrke ${ }^{1}$, \\ Arnulf Dörner ${ }^{2}$ and Matthias Gebauer ${ }^{1}$ \\ ${ }^{1}$ Helios Endo-Klinik Hamburg, Department of Orthopaedic Surgery, Hamburg, Germany \\ ${ }^{2}$ Agaplesion Diakonieklinikum Hamburg, Department of Abdominal Surgery, Hamburg, Germany
}

\begin{abstract}
Background and Purpose: Total hip arthroplasty (THA) is a safe and reliable surgical procedure. However, THA also has intra- and postoperative complications. A dreaded and frustrating intraoperative complication during total hip arthroplasty is dislocation of the femoral trial head from the neck into the pelvis.

Methods: Here, we report on the case of a 71-year old female patient with osteoarthritis of the left hip. Total hip arthroplasty was performed in a lateral position through a standard posterior approach. During intraoperative trial reduction, the femoral trial head dissociated from the taper and dislocated into the psoas compartment. Several unsuccessful attempts, including an additional ventral approach, were made to immediately retrieve the femoral trial head.

Results and interpretation: Postoperative a Computerized Tomography (CT) was performed to locate the trial head, a secondary explorative laparotomy was undertaken to retrieve it. The retrieval of the femoral trial head should be performed in a planned second surgical procedure to avoid possible complications during the manipulation necessary for retrieval.
\end{abstract}

Keywords: Complication, femoral trial head, laparotomy, retrieval, total hip arthroplasty, total hip replacement.

\section{INTRODUCTION}

Total hip arthroplasty (THA) is a safe and reliable surgical procedure for the treatment of painful primary or secondary osteoarthritis of the hip. However, THA also has intra- and postoperative complications. A frustrating intraoperative complication for every surgeon is when the trial femoral head dissociates from the taper and migrates into the pelvis. Making a decision regarding how to deal with such a situation is challenging for every surgeon. As described by other authors, the trial femoral head probably cannot be directly retrieved from the pelvis via only a hip incision [1-4].

We here report on the intraoperative dislocation of the femoral trial head into the iliac muscle during a primary THA.

\section{PATIENT AND CASE DESCRIPTION:}

We report on a 71-year old female patient, admitted to our clinic for elective THA with painful osteoarthritis of the left hip. The examination on admission showed typical clinical signs of progressive osteoarthritis. Radiographs of the left hip showed osteoarthritis. No previous surgeries had been performed. On admission, the body mass index (BMI) of the patient was $23.1 \mathrm{~kg} / \mathrm{m}^{2}(1,61 \mathrm{~m} ; 60 \mathrm{~kg})$. Aside from

*Address correspondence to this author at the Department of Orthopaedic Surgery, Helios Endo Klinik Hamburg, Holstenstrasse 2, D-22767 Hamburg, Germany; Tel: +49 403197 1649; Fax: + 49403197 0;

E-mail:mcitak@gmx.de high blood pressure treated with a single medication and struma nodosa, no other systemic illnesses were noted.

\section{SURGICAL TECHNIQUE}

Surgery was performed by an experienced attending orthopaedic surgeon under spinal anesthesia. After lateral positioning on the operating table, standard preparation and draping was performed. Exposure was performed through a standard posterior approach. Following resection of the femoral head based on the preoperative digital planning, the acetabulum was exposed and reamed. After defining the appropriate cup size (44 mm Mark III cup, Waldemar Link $\mathrm{GmbH}$, Hamburg, Germany) it was implanted using $40 \mathrm{~g}$ Refobacin bone cement. After hardening of the bone cement, remaining osteophytes were removed. Then, the femoral canal was opened and prepared for the implantation of a cemented stem (150mm SP-II stem, Waldemar Link GmbH, Hamburg, Germany). Before final implantation, a trial reduction with a $28 \mathrm{~mm}$ femoral trial head and a trial taper was performed. There was a good range of motion without any signs of impingement and the hip was stable. During removal of the femoral trial head from the cup, the femoral trial head dissociated from the taper and dislocated into the psoas compartment. Several unsuccessful attempts were made to immediately retrieve the femoral trial head. An additional ventral approach was performed, but again remained unsuccessful. Hence, the decision was made to retain the femoral trial head and retrieve it in a planned second surgical procedure. The surgery was continued and the original implants were implanted using a cemented SP-II 
stem (Waldemar Link GmbH, Hamburg, Germany) with a 28 $\mathrm{mm}$ original ceramic head.

After surgery, an anteroposterior radiograph of the pelvis was performed, but the exact location of the femoral trial head was impossible to determine with plain radiographs (Fig. 1). Postoperatively, the situation of the retrieved femoral trial head was discussed with the patient including the options of retention and/or retrieval in situ. After an extended discussion of the possible treatment options, the decision to retrieve the femoral trial head was made together with the patient. First, we performed an additional computerized tomography (CT) of the pelvis to determine the exact location of the trial head in the iliac muscle (Fig. 2).

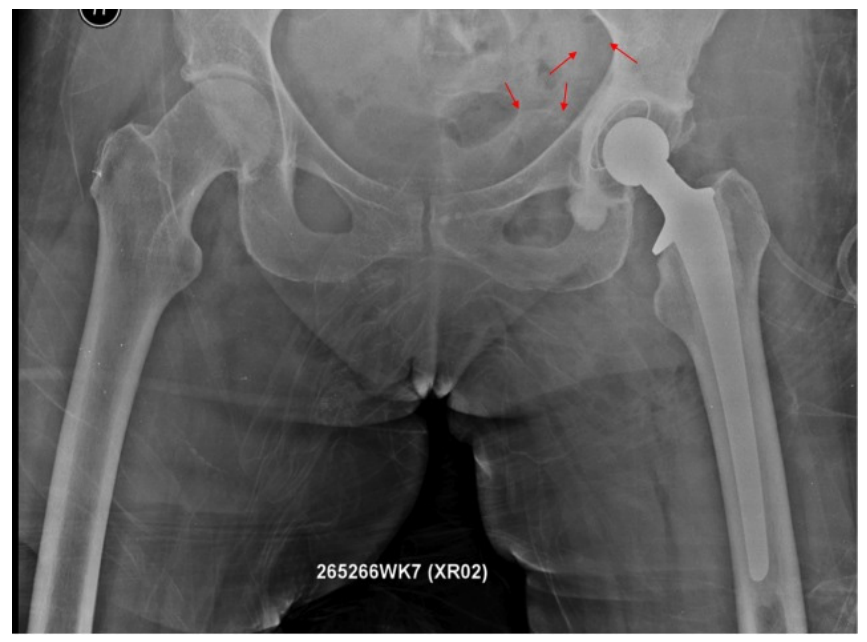

Fig. (1). Postoperative anteroposterior radiograph of the pelvis. Exact localization of the femoral trial head is impossible on plain radiographs.

The patient was then transferred to the department of abdominal surgery for the planned retrieval of the femoral trial head. Surgery was performed by a senior abdominal surgeon. After positioning on the operating table in the supine position, standard preparation and draping was performed. The femoral trial head was retrieved out of the iliac muscle via an extraperitoneal approach at the lower left abdomen (Fig. 3). Sixteen days after primary THA, the patient was finally discharged without further complications.

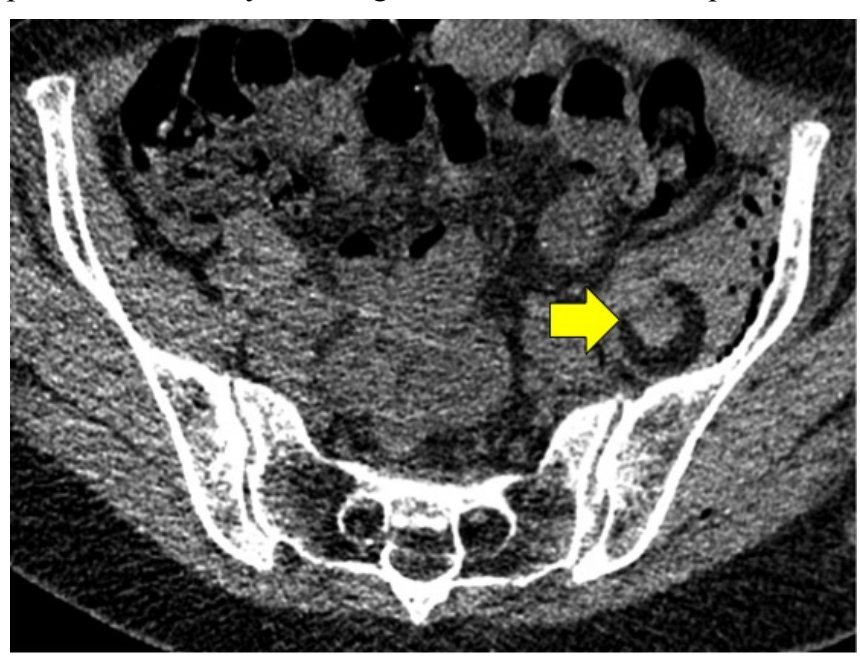

Fig. (2). Computerized Tomography of the pelvis allows the exact localization of the femoral trial head into the iliac muscle.

\section{DISCUSSION}

Minimally invasive total hip arthroplasty (MITHA) has gained popularity in recent years [5]. Described advantages of MITHA include less soft-tissue trauma due to a smaller skin incision and less muscle damage, reduced blood loss, shorter hospital stay, less pain and faster recovery [6-8].

However, Alfonso et al. concluded that the use of a small incision approach for hip arthroplasty may lead to an increased prevalence of femoral trial head dislocation due to

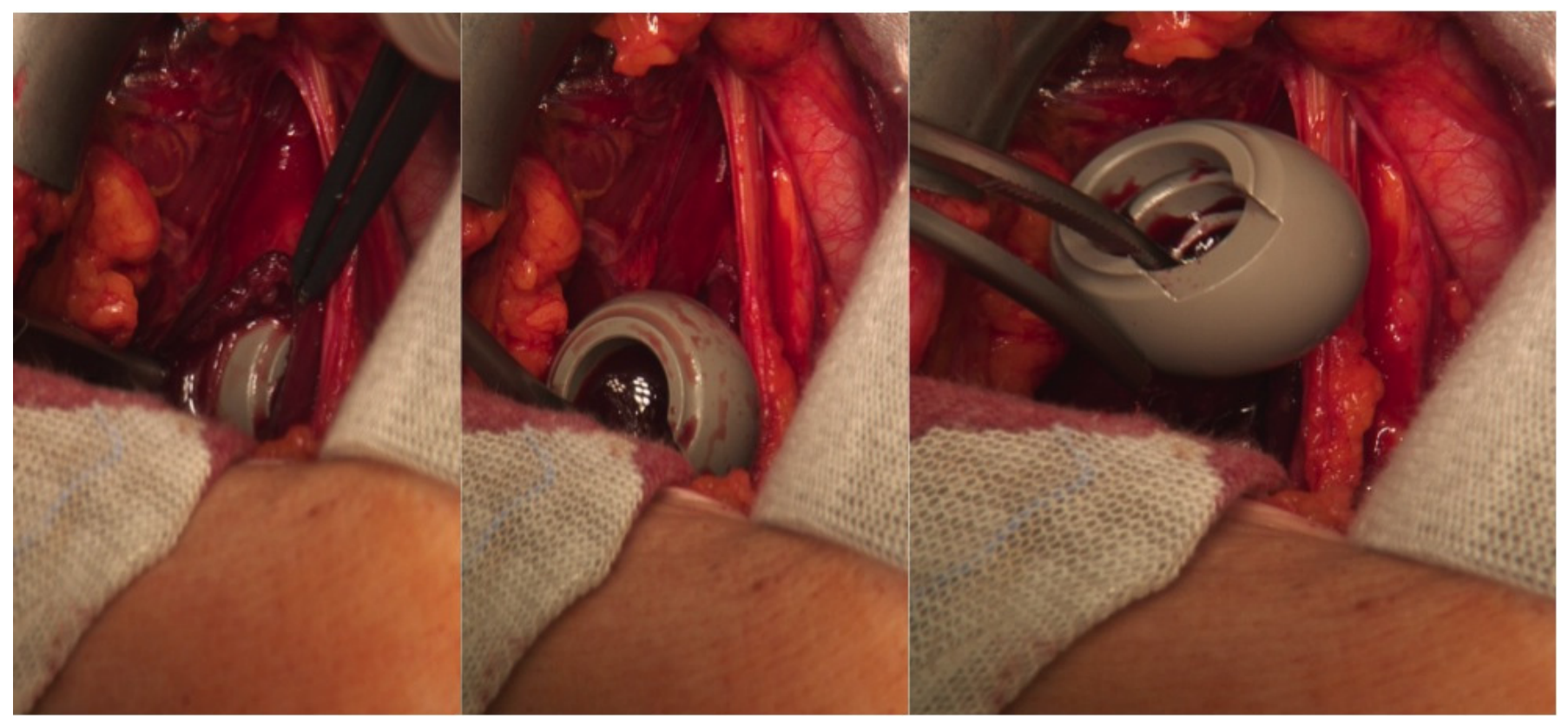

Fig. (3). The femoral trial head was detected after performing a exploratory laparotomy in the musculus iliacus and was retrieved without any complications using a clamp. 
the limited size of the surgical field [9]. In this case, several attempts to retrieve the femoral trial head were unsuccessful. Postoperatively, the patient complained of pain in the lower abdominal quadrants. A laparoscopic surgery was performed to retrieve the femoral trial head [9]. Alfonso et al. described a technique regarding how to avoid the loosening of the femoral trial head during THA [9]. Their technique consists of threading two heavy sutures through the dome holes of the trial femoral head and securing the loop with a figure-ofeight knot [9]. As described by Alfonso et al. and other authors, the trial femoral head probably cannot be retrieved from the pelvis through only a hip incision [1-4]. Making decisions regarding how to deal with such situations is challenging for every orthopaedic surgeon.

Vertelis et al. reported on femoral trial head retention in a 76-year old female patient [10]. They performed a control CT scan of the pelvis 8 months after surgery, where the head was found in the same place as in the previous CT scan and no reactions of the surrounding tissues were found [10].

Callaghan reported on a similar case of a 67-year old male patient in a relatively poor condition. Two years postoperatively, the patient had no symptoms with the retained femoral trial head [2].

Batouk et al. described a case of femoral trial head retention in a 81-year-old male patient [11]. Three months after surgery, the patient did not report any notable pain in the right hip or the pelvis [11].

To the best of our knowledge, three reported cases of dislocated femoral trial head retention exist to date. As of the latest follow-up, none of the reported cases had developed a complication and all were pain-free. Despite the successful reported cases of femoral trial head retention, we decided to retrieve the femoral trial head during a secondary intervention after a thorough discussion of the situation with the patient.

\section{CONCLUSIONS}

In summary, the decision regarding retrieval or retention of the femoral trial head is challenging in such a situation. Fixation of the femoral trial head with two heavy sutures may avoid loosening of the trial head during trial reduction. We recommend, in such a situation, to perform an additional radiographic examination postoperatively, including a CT scan to detect the exact location of the femoral trial head.
The retrieval of the femoral trial head should be performed in a planned second surgical procedure to avoid possible complications during the manipulation that is necessary for removal. This case underscores the necessity of further innovations for more stabile head-and-neck trial components for primary or revision THA.

\section{CONFLICT OF INTEREST}

The authors confirm that this article content has no conflict of interest.

\section{ACKNOWLEDGEMENTS}

Declared none.

\section{REFERENCES}

[1] Ziv YB, Backstein D, Safir O, et al. Intraoperative dislocation of a trial femoral head into the pelvis during total hip arthroplasty. Can J Surg 2008; 51(3): E73-4

[2] Callaghan JJ, McAndrew C, Boese CK, et al. Intrapelvic migration of the trial femoral head during total hip arthroplasty: is retrieval necessary? A report of four cases. Iowa Orthop J 2006; 26: 60-2.

[3] Rachbauer F, Nogler M, Krismer M, et al. Intraoperative migration of the trial femoral head into the pelvis during total hip arthroplasty: prevention and retrieval. J Bone Joint Surg Am 2002; 84-A(5): 881-2; author reply 882 .

[4] Princep A. Intraoperative migration of the trial femoral head into the pelvis during total hip arthroplasty: prevention and retrieval. J Bone Joint Surg Am 2002; 84-A(5): 880-1; author reply 881.

[5] Berry DJ, Berger RA, Callaghan JJ, et al. Minimally invasive total hip arthroplasty. Development, early results, and a critical analysis. Presented at the Annual Meeting of the American Orthopaedic Association, Charleston, South Carolina, USA, June 14, 2003. J Bone Joint Surg Am 2003; 85-A(11): 2235-46.

[6] Sculco TP, Jordan LC, Walter WL. Minimally invasive total hip arthroplasty: the Hospital for Special Surgery experience. Orthop Clin North Am 2004; 35(2): 137-42.

[7] Wall SJ, Mears SC. Analysis of published evidence on minimally invasive total hip arthroplasty. J Arthroplasty 2008; 23(7 Suppl): $55-8$

[8] Pagnano MW, Hebl J, Horlocker T. Assuring a painless total hip arthroplasty: a multimodal approach emphasizing peripheral nerve blocks. J Arthroplasty 2006; 21(4 Suppl 1): 80-4.

[9] Alfonso D, Idjadi J, Lamont JG. Retrieval of a trial femoral head that displaces into the periacetabular soft tissue during miniincision total hip arthroplasty. A case report. J Bone Joint Surg Am 2006; 88(4): 866-8.

[10] Vertelis A, Vertelis L, Tarasevicius S. Trial femoral head loss in to the soft tissues of pelvis during primary total hip replacement: a case report. Cases J 2008; 1(1): 151.

[11] Batouk O, Gilbart M, Jain R. Intraoperative dislocation of the trial femoral head into the pelvis during total hip arthroplasty: a case report. J Bone Joint Surg Am 2001; 83-A(10): 1549-51. 